\title{
Does surface roughness amplify wetting?
}

\author{
Alexandr Malijevský \\ Department of Physical Chemistry, Institute of Chemical Technology, Prague, 16628 Praha 6, Czech Republic \\ Institute of Chemical Process Fundamentals, Academy of Sciences, 16502 Prague 6, Czech Republic
}

\begin{abstract}
Any solid surface is intrinsically rough on the microscopic scale. In this paper, we study the effect of this roughness on the wetting properties of hydrophilic substrates. Macroscopic arguments, such as those leading to the well-known Wenzel's law, predict that surface roughness should amplify the wetting properties of such adsorbents. We use a fundamental measure density functional theory (DFT) to demonstrate the opposite effect from roughness for microscopically corrugated surfaces, i.e., wetting is hindered. Based on three independent analyses we show that microscopic surface corrugation increases the wetting temperature or even makes the surface hydrophobic. Since for macroscopically corrugated surfaces the solid texture does indeed amplify wetting there must exist a crossover between two length-scale regimes that are distinguished by opposite response on surface roughening. This demonstrates how deceptive can be affords to extend the thermodynamical laws beyond their macroscopic territory.
\end{abstract}

\section{INTRODUCTION}

It is well known that the geometric or chemical inhomogeneity of a solid surface can dramatically change its adsorption properties [1-3]. For instance, even the most hydrophobic surfaces that are considered smooth on the macroscopic scale, can be characterized by the contact angle of a sessile liquid drop that typically does not substantially exceed $\theta \approx 120^{\circ}$. The equilibrium contact angle is defined by Young's equation [4]

$$
\gamma_{\mathrm{sv}}=\gamma_{\mathrm{sl}}+\gamma \cos \theta \text {, }
$$

in terms of the tensions of the solid-vapor, solid-liquid and liquid-vapor interfaces. However, if the same material is textured, i.e. the surface is rough, the micro- or nanostructure of the surface may induce contact angles close to $180^{\circ}$. This effect has important applications in modern technologies for the fabrication of devices that utilize super-hydrophobic surfaces. Advances in these technologies are often inspired by natural self-cleaning properties [5] of certain plant leaves and incest wings where the combination of hydrophobicity and roughness leads to the Lotus effect where dirt particles are adsorbed and removed by water droplets rolling off these surfaces [6. These super-hydrophobic surfaces are replicated in nanotechnologies by grafting polymer chains onto a substrate [7] or using nanoimprint lithography [8].

Macroscopically, the effect of roughness on substrate wettability is often expressed in terms of the effective (or apparent) contact angle $\theta^{*}$, which corresponds to the equilibrium configuration of a macroscopic liquid drop sitting on a rough surface. One of the most popular relations between Young's and effective contact angles provides Wenzel's law [9]: assuming that a liquid is in complete contact with a rough surface, i.e., the liquid enters into the grooves of the surface beneath the drop, simple thermodynamic arguments dictate that:

$$
\cos \theta^{*}=r \cos \theta,
$$

where $r>1$ is a roughness parameter defined as the ratio of the actual area of the solid surface to the normally projected area.

The most important qualitative conclusion that can be drawn from the Wenzel equation is that surface roughness always amplifies the wetting properties of a given surface. Therefore, surface roughness makes hydrophobic surfaces (or, more generally, surfaces exhibiting partial drying toward a given fluid) even more hydrophobic, i.e., $\theta^{*}>\theta>90^{\circ}$, in line with the aforementioned examples of super-hydrophobicity. However, hydrophilic surfaces (or, more generally, surfaces exhibiting partial wetting toward a given fluid) are predicted to be rendered hydrophilic still more by surface roughness, since $\theta^{*}<\theta<90^{\circ}$, according to Eq. (2).

It is considerably more challenging to develop a description of substrate topography effects at the microscopic scale. On the molecular level, the competition between the fluid-fluid and the fluid-wall interactions, as well as the detailed structure of the wall must be properly considered. While wetting phenomena on structureless substrates are fairly well understood [10 13, a connection between adsorption on microscopically corrugated surfaces and the underlying intermolecular forces is still largely missing. One obvious reason for the absence of such a description is that in contrast with structureless walls, that present a one-dimensional problem [14, the two- or three-dimensional problem of rough surfaces is much more involved computationally, such that the literature involving theoretical [15-20] and simulation 21-23 studies is rather limited.

In this paper, we study the wetting behavior of rough solid surfaces using a microscopic density functional theory (DFT) 24. In particular, we determine how the surface roughness affects the wetting properties of a hydrophilic substrate. Our DFT is based on the Tarazona version [25, 26] of the fundamental measure theory (FMT) [27, which accurately captures short-range correlations between particles and satisfies exact statistical mechanical sum rules. The rough substrate is modeled as a semi-infinite planar wall onto which a linear array of tiny pillars is deposited. Translation symmetry along one 
Cartesian dimension is assumed and gravity is ignored. The model is similar to the one considered in Ref. 28 for a grooved substrate, but differs in several aspects: i) The corrugation of the wall is much finer and represents an intrinsic surface roughness rather than a fabricated structure. Thus, capillary effects inside the grooves between two neighboring pillars play only a minor role and the dimension of these grooves cannot induce a filling transition. Therefore, the only relevant surface phase transition is the wetting transition, when the contact angle of a sessile drop vanishes. We particularly wish to determine how the temperature at which the wetting transition occurs depends on the wall geometry. ii) In Ref. [28], the dispersion force exerted by the wall was induced by the interaction between solid atoms and fluid atoms via the attractive part of the Lennard-Jones potential. The repulsive part of the interaction was modeled by coating the outer layer of the solid atoms with a hard wall of a thickness corresponding to the fluid atom diameter. Such a model somewhat exaggerates the repulsion near the apex of rectangular ridges, which in turn overestimates the attraction of the interfacial potential that binds the wetting film to the substrate due to the edge-shaped geometry of the substrate [29, 30]. In this study, the interaction between the wall and fluid atoms is described by the full Lennard-Jones potential avoiding the need to introduce an extra hard-wall repulsion, which makes a description of the fluid structure near the edges more realistic. iii) In our previous studies, we described the strongly oscillating structure of the fluid that is induced by strong confinement using the original Rosenfeld's FMT [27. In the present study, we employ a more sophisticated version of the FMT involving tensorial weighted densities that ensure the correct performance of the resulting functional in reduced dimensions, which is important for strongly confining geometries. This functional is probably the most satisfactory description of packing effects within the currently available approximations of the intrinsic free energy for nonuniform fluids.

The primary result of this study is that, contrary to macroscopic predictions, the microscopic surface roughness always deteriorates the substrate wettability. We demonstrate this phenomenon using three different numerical DFT analyzes: 1) We use a standard grandcanonical ensemble DFT to determine the wetting temperature $T_{w}$ and the contact angle temperature dependence for $T<T_{w}$. 2) We use a constrained DFT (cDFT) where we fix the average number of particles per unit length to determine the equilibrium shape of the liquidvapor interface. 3) Finally, we contrast the wetting behavior of smooth and rough surfaces by considering a substrate which is an assemble of both types of surfaces. Using both DFT and cDFT we demonstrate that wetting of the rough sections is disfavored until a relatively high temperature that correspond to the wetting temperature of the rough section, when the entire surface becomes completely wet.

Before concluding this section we want to emphasize

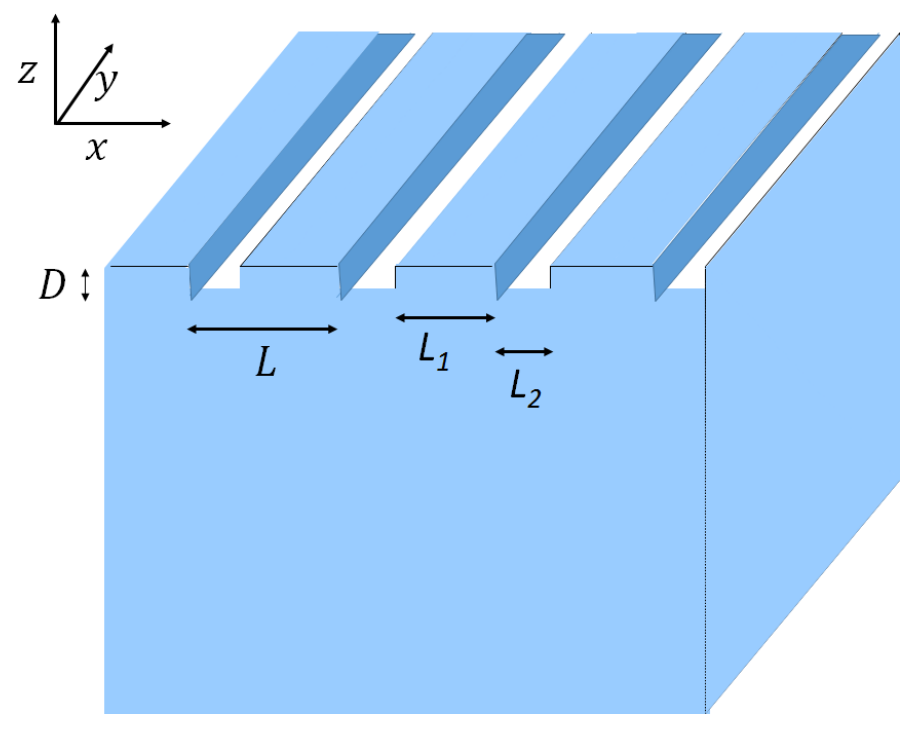

FIG. 1: Schematic picture of our model of a rough surface. The pillars of height $D$ and width $L_{1}$ are deposited on a semiinfinite slab with a periodicity $L$. The model is assumed to be translation invariant along the $y$-axis.

that the intention of this work is not to scrutinize the limit of validity of Wenzel's relation Eq. (2) on the macroscopic scale, as has already been done in numerous studies (see, e.g., Refs. [3, 31 34]). Instead, we want to highlight that any approach based solely on phenomenological arguments fails to account for even a qualitative description of wetting for microscopically rough surfaces. This failure can particularly well be illustrated on the simple Eq. (2) but it is no specific feature of the Wenzel's relation. We will come back to this point in the final section.

The remainder of the paper is organized as follows. In the following section, we define the microscopic model and formulate the DFT and cDFT treatments. The numerical results are presented in section III: we first describe the wetting properties of a smooth wall and then contrast these results with the wetting properties of corrugated substrates. The results are summarized in section IV and discussed in the concluding section V.

\section{DENSITY FUNCTIONAL THEORY}

\section{A. Grand canonical ensemble DFT}

Within the classical density functional theory formulated by Evans [24, the equilibrium density profile is determined by minimizing the grand potential functional:

$$
\Omega[\rho]=\mathcal{F}[\rho]+\int \mathrm{d} \mathbf{r} \rho(\mathbf{r})[V(\mathbf{r})-\mu],
$$

where $\mu$ is the chemical potential and $V(\mathbf{r})$ is the external potential. The intrinsic free energy functional $\mathcal{F}[\rho]$ can 
be divided into an exact ideal gas contribution and an excess part:

$$
\mathcal{F}[\rho]=\frac{1}{\beta} \int \mathrm{d} \mathbf{r} \rho(\mathbf{r})\left[\ln \left(\rho(\mathbf{r}) \Lambda^{3}\right)-1\right]+\mathcal{F}_{\mathrm{ex}}[\rho],
$$

where $\Lambda$ is the thermal de Broglie wavelength, which can be set to unity and $\beta=1 / k_{B} T$ is the inverse temperature. Following a standard perturbation approach, the excess term is further split into hard-sphere and attractive contributions where the latter is treated in a simple mean-field fashion. Thus we write

$$
\mathcal{F}_{\mathrm{ex}}[\rho]=\mathcal{F}_{\mathrm{hs}}[\rho]+\frac{1}{2} \iint \mathrm{d} \mathbf{r} \mathrm{d} \mathbf{r}^{\prime} \rho(\mathbf{r}) \rho\left(\mathbf{r}^{\prime}\right) u_{\mathrm{a}}\left(\left|\mathbf{r}-\mathbf{r}^{\prime}\right|\right),
$$

where $u_{\mathrm{a}}(r)$ is the attractive part of the fluid-fluid interaction potential.

In our model, the fluid atoms are assumed to interact with each other via a truncated (but non-shifted) Lennard-Jones-like potential

$$
u_{\mathrm{a}}(r)=\left\{\begin{array}{cc}
0 ; & r<\sigma \\
-4 \varepsilon\left(\frac{\sigma}{r}\right)^{6} ; & \sigma<r<r_{c}, \\
0 ; & r>r_{c} .
\end{array}\right.
$$

which is cut-off at $r_{c}=2.5 \sigma$, where $\sigma$ is the hard-sphere diameter. Hereafter, we will use the parameters $\sigma$ and $\varepsilon$ as the length and energy units.

The hard-sphere part of the excess free energy is approximated by the fundamental measure theory (FMT) functional [27]:

$$
\mathcal{F}_{\mathrm{hs}}[\rho]=\frac{1}{\beta} \int \mathrm{d} \mathbf{r} \Phi\left(\left\{n_{\alpha}\right\}\right) .
$$

Within the original Rosenfeld's approach, the weighted densities $n_{\alpha}$ consist of four scalar and two vector functions, which are given by convolutions of the density profile and the corresponding weight function:

$$
n_{\alpha}(\mathbf{r})=\int \mathrm{d} \mathbf{r}^{\prime} \rho\left(\mathbf{r}^{\prime}\right) w_{\alpha}\left(\mathbf{r}-\mathbf{r}^{\prime}\right) \quad \alpha=\{0,1,2,3, v 1, v 2\}
$$

where $w_{3}(\mathbf{r})=\Theta(R-|\mathbf{r}|), w_{2}(\mathbf{r})=\delta(R-|\mathbf{r}|), w_{1}(\mathbf{r})=$ $w_{2}(\mathbf{r}) / 4 \pi R, w_{0}(\mathbf{r})=w_{2}(\mathbf{r}) / 4 \pi R^{2}, w_{v 2}(\mathbf{r})=\frac{\mathbf{r}}{R} \delta(R-|\mathbf{r}|)$, and $w_{v 1}(\mathbf{r})=w_{v 2}(\mathbf{r}) / 4 \pi R$. Here, $\Theta(r)$ is the Heaviside function, $\delta(r)$ is the Dirac delta function and the hardsphere radius was set to $R=\sigma / 2$.

It is well known that the original Rosenfeld's functional and all of the alternative approaches that use the set of weighted densities according to (8) provide an excellent description of short range correlations and satisfy the exact statistical mechanical sum rules and thermodynamic conditions at planar walls and corners 35. However, this class of functionals fails to describe the hard-sphere crystal and can produce spurious divergences for highly packed systems beyond the planar geometry [36]. Here, we use the FMT version proposed by Tarazona [25, 26] in which the set of weighted densities (8) is complemented by a tensor density with Cartesian components:

$$
T_{i j}(\mathbf{r})=\int \mathrm{d} \mathbf{r}^{\prime} \rho\left(\mathbf{r}+\mathbf{r}^{\prime}\right) \frac{r_{i}^{\prime} r_{j}^{\prime}}{R^{2}} \delta\left(R-\left|\mathbf{r}^{\prime}\right|\right)
$$

The free-energy density is then given by

$$
\begin{aligned}
\Phi & =-n_{0} \ln \left(1-n_{3}\right)+\frac{n_{1} n_{2}-\mathbf{n}_{v 1} \cdot \mathbf{n}_{v 2}}{1-n_{3}} \\
& +\frac{3}{16 \pi} \frac{\mathbf{n}_{v 2} \cdot \mathbf{T} \cdot \mathbf{n}_{v 2}-n_{2} n_{v 2}^{2}-\operatorname{Tr}\left[\mathbf{T}^{3}\right]+n_{2} \operatorname{Tr}\left[\mathbf{T}^{2}\right]}{\left(1-n_{3}\right)^{2}}
\end{aligned}
$$

where $\mathbf{T}$ is the matrix corresponding to $(9)$ and where we have kept the original Rosenfeld's notation.

Minimizing Eq. (3) yields an Euler-Lagrange equation:

$\frac{1}{\beta} \ln \Lambda^{3} \rho(\mathbf{r})+\frac{\delta \mathcal{F}_{\mathrm{hs}}[\rho]}{\delta \rho(\mathbf{r})}+\int \mathrm{d} \mathbf{r}^{\prime} \rho\left(\mathbf{r}^{\prime}\right) u_{\mathrm{a}}\left(\left|\mathbf{r}-\mathbf{r}^{\prime}\right|\right)=\mu-V(\mathbf{r})$.

An external potential $V(\mathbf{r})$ representing a corrugated wall, is constructed by considering a semi-infinite solid slab of uniform density $\rho_{w}$ into which an infinite array of infinitely long narrow grooves is cut, as illustrated in Fig. 1. Alternatively, the wall surface can be viewed as a planar wall onto which an infinite array of infinitely long pillars is deposited. The depth of the grooves or the height of the pillars is $D$. The width of the pillars is $L_{1}$ and the width of the grooves is $L_{2}$, such that $L=$ $L_{1}+L_{2}$ is the periodicity of the potential. The wall atoms interact with the fluid particles via the LennardJones potential

$$
\phi(r)=4 \varepsilon_{w}\left[\left(\frac{\sigma}{r}\right)^{12}-\left(\frac{\sigma}{r}\right)^{6}\right],
$$

such that total external potential experienced by the fluid atoms is

$$
V(x, z)=V_{\pi}(z)+\sum_{n=-\infty}^{\infty} V_{p}(x+n L, z),
$$

where

$$
V_{\pi}(z)=4 \pi \varepsilon_{w} \rho_{w} \sigma^{3}\left[\frac{1}{45}\left(\frac{\sigma}{z}\right)^{9}-\frac{1}{6}\left(\frac{\sigma}{z}\right)^{3}\right]
$$

is the potential of the flat wall and $V_{p}(x, z)$ is the potential of a single pillar:

$$
\begin{aligned}
V_{p}(x, z)= & \rho_{w} \int_{0}^{L_{1}} \mathrm{~d} x^{\prime} \int_{-\infty}^{\infty} \mathrm{d} y \int_{0}^{D} \mathrm{~d} z^{\prime} \\
& \phi\left(\sqrt{\left(x-x^{\prime}\right)^{2}+y^{2}+\left(z-z^{\prime}\right)^{2}}\right) \\
\equiv & V_{12}(x, z)+V_{6}(x, z) .
\end{aligned}
$$

The $V_{12}(x, z)$ term describes the repulsive part of the wall-fluid interaction and has the form:

$$
\begin{aligned}
V_{12}(x, z)= & 4 \varepsilon_{w} \sigma^{12} \rho_{w} \int_{x-L_{1}}^{L_{1}} \mathrm{~d} x^{\prime} \int_{-\infty}^{\infty} \mathrm{d} y \int_{z-D}^{z} \mathrm{~d} z^{\prime} \frac{1}{\left(x^{\prime 2}+y^{2}+z^{\prime 2}\right)^{6}} \\
= & \pi \varepsilon_{w} \sigma^{12} \rho_{w}\left[\psi_{12}(x, z)-\psi_{12}(x, z-D)\right. \\
& \left.-\psi_{12}\left(x-L_{1}, z\right)+\psi_{12}\left(x-L_{1}, z-D\right)\right]
\end{aligned}
$$

where 
$\psi_{12}(x, z)=-\frac{1}{2880} \frac{128 x^{16}+448 x^{14} z^{2}+560 x^{12} z^{4}+280 x^{10} z^{6}+35 x^{8} z^{8}+280 x^{6} z^{10}+560 x^{4} z^{12}+448 z^{14} x^{2}+128 z^{16}}{z^{9} x^{9}\left(x^{2}+z^{2}\right)^{7 / 2}}$

The attractive contribution from a single pillar can be conveniently written as 28]

$$
\begin{aligned}
V_{6}(x, z)= & -4 \varepsilon_{w} \sigma^{6} \rho_{w} \int_{x-L_{1}}^{L_{1}} \mathrm{~d} x^{\prime} \int_{-\infty}^{\infty} \mathrm{d} y \int_{z-D}^{z} \mathrm{~d} z^{\prime} \\
& \frac{1}{\left(x^{\prime 2}+y^{2}+z^{\prime 2}\right)^{3}} \\
= & \alpha_{w}\left[\psi_{6}(x, z)-\psi_{6}(x, z-D)\right. \\
& \left.-\psi_{6}\left(x-L_{1}, z\right)+\psi_{6}\left(x-L_{1}, z-D\right)\right](18)
\end{aligned}
$$

where

$$
\alpha_{w}=-\frac{1}{3} \pi \varepsilon_{w} \sigma^{6} \rho_{w}
$$

and

$$
\psi_{6}(x, z)=-\frac{2 x^{4}+x^{2} z^{2}+2 z^{4}}{2 z^{3} x^{3} \sqrt{x^{2}+z^{2}}} .
$$

Having determined the equilibrium density profile $\rho(x, z)$ we construct the excess adsorption

$$
\Gamma=\frac{1}{L} \iint \mathrm{d} x \mathrm{~d} z\left(\rho(x, z)-\rho_{b}\right),
$$

where $\rho_{b}$ is the density of the bulk phase and the integral is performed over one period of the system accessible volume. The excess adsorption serves as an order parameter for determining the wetting temperature $T_{w}$ and is finite for $T<T_{w}$ and diverges for $T \geq T_{w}$.

\section{B. Constrained DFT}

In the previous paragraph a standard grand-canonical DFT was presented. In order to describe a shape of a cylindrical drop deposited on a smooth or rough surface we also employ what we call here constrained DFT, in which case the average number of particles, rather than directly the chemical potential, is maintained fixed. Owing to the translation symmetry of the (infinite) system along one of the directions parallel with the wall, we fix

$$
\int \mathrm{d} x \mathrm{~d} z \rho(x, z)=\frac{\langle N\rangle}{L_{\|}},
$$

where the r.h.s. of Eq. (22) expresses the average number of particles per unit length of the system in the $y$ direction.

We note that the computation of the equilibrium density profiles is still performed in a grand-canonical ensemble using the same grand-canonical intrinsic free energy functional (5) as in the previous case, such that we minimize

$$
\mathcal{F}[\rho]+\int \mathrm{d} \mathbf{r} \rho(\mathbf{r}) V(\mathbf{r}),
$$

subject to the constraint of Eq. 22. This approach should be distinguished from a canonical ensemble DFT describing closed systems, i.e. those at which the (integer) number of particles $N$, rather than the average number of particles $\langle N\rangle$, is fixed. This canonical ensemble DFT can be performed either using a free energy functional derived in the formalism of the canonical ensemble [37] or by linking approximately the grand canonical DFT functional with the canonical ensemble one [38 40]. Neither is necessary for our purposes where we deal with open systems with a large number of particles (in contrast to systems containing only few particles that are confined by a closed impenetrable cavity as considered in Refs. [38 40]). For systems far from any phase transition, this approach is completely equivalent to the standard unconstrained DFT as the constraint imposed by Eq. (22) is equivalent to fixing a corresponding value of the chemical potential. However, a special care must be taken for systems at or near-to two-phase equilibrium. In these cases, the constrained DFT allows to stabilize a mixture of these two phases due to the constraint of the system but a reasonable initial conditions respecting the system symmetry must be imposed in order to avoid artificial results.

The constrained minimization leads to the following equation for the equilibrium density profile:

$$
\rho(\mathbf{r})=\frac{N \exp \left[c^{(1)}(\mathbf{r})-\beta V(\mathbf{r})\right]}{\int \mathrm{d} \mathbf{r} \exp \left[c^{(1)}(\mathbf{r})-\beta V(\mathbf{r})\right]},
$$

where

$$
c^{(1)}(\mathbf{r})=-\frac{\delta F_{\mathrm{ex}}[\rho(\mathbf{r})] / k_{B} T}{\delta \rho(\mathbf{r})}
$$

is the grand canonical single-particle direct correlation function. Eq. (24) can be solved iteratively by starting from an initial density profile $\rho(x, z)$, such that a part of the box (presumably to be occupied by the liquid drop) has a uniform density $\rho_{l}(T)$ and the rest of the box is of a uniform density $\rho_{v}(T)$. Upon iterating Eq. (24) the density profile evolves toward the equilibrium such that the entire average density of the system remains unchanged.

Both Euler-Lagrange equations (11) and 24, corresponding to DFT and cDFT, respectively, are solved using the Picard iteration for the equilibrium profile $\rho(x, z)$ on a two-dimensional Cartesian grid with a spacing of 
$0.05 \sigma$. After an appropriate transformation of the coordinate system, the corresponding two-dimensional integrals are expressed over the interval $(-1,1)$ and approximated by the Gaussian quadrature. The value of the integrands at the points out of the grid is evaluated by the bilinear interpolation, see Ref. [28] for more details.

\section{RESULTS}

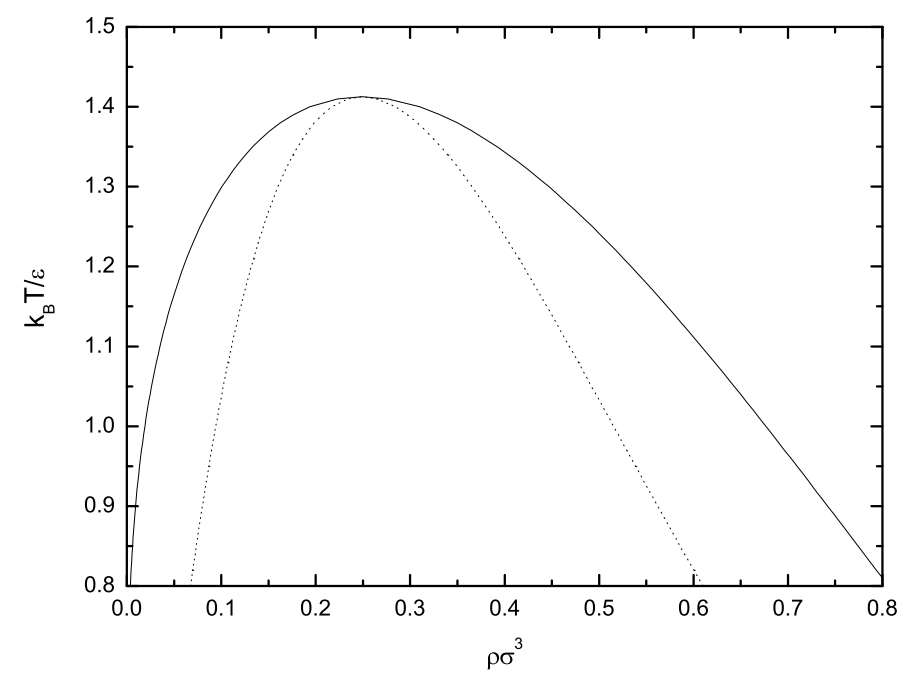

FIG. 2: Bulk phase diagram for a model fluid consisting of a hard-sphere repulsion (over the range of $\sigma$ ) and a truncated $\left(r_{c}=2.5 \sigma\right)-4 \varepsilon \sigma^{6} / r^{6}$ attraction; the solid line represents vapor-liquid coexistence (binodal), and the dotted line represents the limit-of-stability (spinodal).

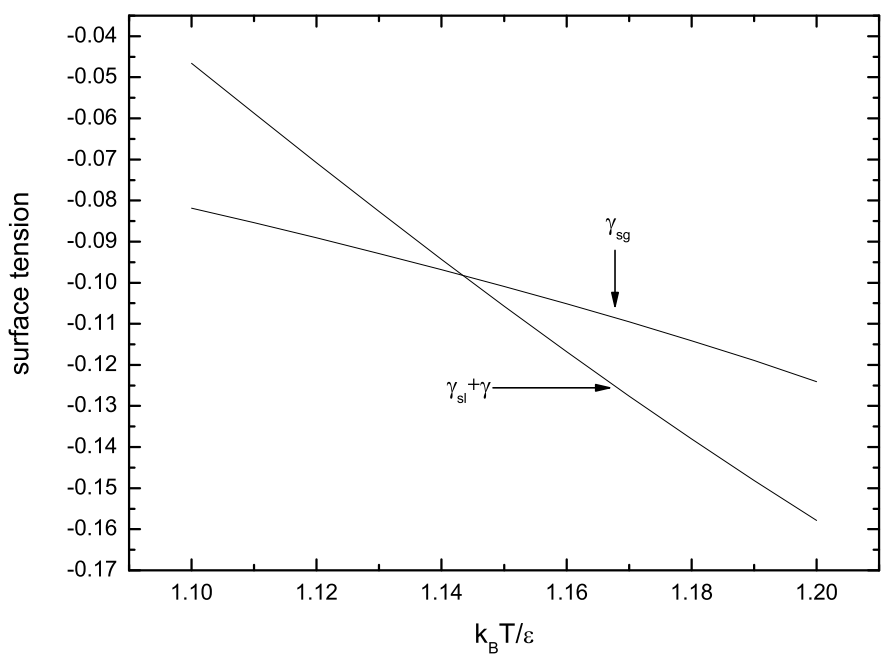

FIG. 3: Determination of the wetting temperature $\left(k_{B} T_{w} \approx\right.$ $1.142 \varepsilon$ ) for a planar wall from the intersection of the solidvapor surface tension $\gamma_{s g}$ with the summed tensions $\gamma_{s l}+\gamma$; surface tensions are expressed in units of $\varepsilon / \sigma^{2}$.

In this section, we present numerical results of the
DFT model that was described in the previous section. Before analyzing the surface properties of the model substrates, we present the bulk properties of our model fluid, using Eq. (11) for the case of zero external field. In Fig. 2, we show the liquid-vapor phase diagram in the temperature-density plane, delineating the phase boundary and the limit-of-stability. The latter is defined by the condition $\frac{\partial^{2}(F / V)}{\partial \rho^{2}}=0$ and determines the region of the phase diagram where thermodynamic states cease to be even locally stable. The two curves terminate at a critical point, which has coordinates $k_{B} T_{c}=1.411 \varepsilon$ and $\rho_{c}=0.249 \sigma^{-3}$, as determined by the additional condition $\frac{\partial^{3}(F / V)}{\partial \rho^{3}}=0$.

\section{A. Wetting on a planar wall}

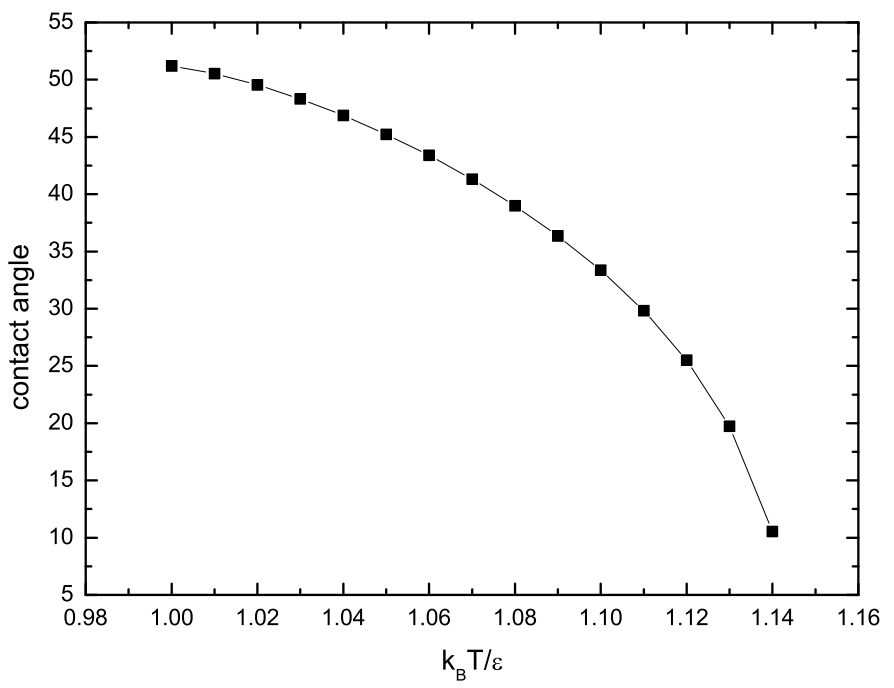

FIG. 4: Temperature dependence of the contact angle (in degrees) of a sessile drop resting on an ideally planar wall.

To begin, we examine the wetting properties of a "reference system" of a planar wall with the potential given by Eq. (14). This system corresponds to a onedimensional problem in which the equilibrium density profile varies in a single Cartesian coordinate, $\rho(\mathbf{r})=$ $\rho(z)$. Nevertheless, for numerical consistency, the system is treated in the same manner as the models of the corrugated walls, in which the external potential, and thus the equilibrium density profile, is two-dimensional. The comparison between the results from one-dimensional and two-dimensional treatments of the system revealed a very good agreement which provides good verification of our numerical methods.

Throughout the study the wall strength is fixed at $\varepsilon_{w}=\varepsilon$. As shown below, the wetting temperature of the flat wall is well below the bulk critical temperature for this value of the wall parameter, which avoids potential complications from critical fluctuations near $T_{c}$ in 


\begin{tabular}{cccc}
\hline case No. & model parameters & $r$ & contact angle \\
\hline 1 & planar wall & 1 & 28 \\
2 & $D=0.5 \sigma, L=4 \sigma$ & 1.25 & 58 \\
3 & $D=0.5 \sigma, L=2 \sigma$ & 1.5 & 77 \\
4 & $D=1.0 \sigma, L=4 \sigma$ & 1.5 & 80 \\
5 & $D=2.0 \sigma, L=4 \sigma$ & 2 & 108 \\
6 & $D=1.0 \sigma, L=2 \sigma$ & 2 & 116 \\
7 & $D=2.0 \sigma, L=2 \sigma$ & 3 & 129 \\
\hline
\end{tabular}

TABLE I: Contact angles at a temperature corresponding to $k_{B} T=1.1 \varepsilon$ for walls with different types of corrugation; the corrugation is formed by pillars of depth $D$, width $L_{1}=0.5 \sigma$ and a periodicity $L$; in the second column, the "surface roughness" of each of the models is estimated as $r \approx 1+2 D / L$; in the third column, the contact angle of the sessile drop is expressed in degrees.

view of the mean-field nature of DFT. On the other hand, this value of $\varepsilon_{w}$ is sufficiently small for the system to exhibit layering transitions, the study of which is beyond the scope of this work.

Perhaps the most straightforward approach to determine the wetting temperature $T_{w}$ at which the thickness of the adsorbed liquid film diverges (and the contact angle of the sessile drop vanishes) is to use Young's equation (1), which transforms to Antonoff's rule $\gamma_{\mathrm{sv}}\left(T_{w}\right)=$ $\gamma_{\mathrm{sl}}\left(T_{w}\right)+\gamma\left(T_{w}\right)$ exactly at $T_{w}$. The surface tensions $\gamma_{\mathrm{sv}}$ and $\gamma_{\mathrm{sl}}$ are obtained by minimizing the grand potential functional given in Eq. (3) subject to the boundary conditions $\rho\left(x, z_{M}\right)=\rho_{v}$ and $\rho\left(x, z_{M}\right)=\rho_{l}, \forall x$, respectively, where $\rho_{v}$ and $\rho_{l}$ are the coexisting densities and $z_{M}$ is the size of the system in the $z$-dimension. Of course, for a planar wall, the density profile $\rho(x, z)$ does not depend on the $x$-coordinate (along the wall); additional periodic boundary conditions $\rho(0, z)=\rho\left(x_{M}, z\right), \forall z$, are imposed for the corrugated walls that are considered in the following paragraph, with $x_{M}$ being the size of the system in the $x$-dimension. The liquid-vapor surface tension $\gamma$ is determined independently by equilibrating a system filled with the two coexisting fluid phases in the absence of an external field. Here, we verify again that both numerical DFT treatments produce consistent results.

In Fig. 3, we show the temperature dependence of the solid-vapor surface tension $\gamma_{s v}$ and the summed tensions $\gamma_{s l}+\gamma$. The intersection of the two curves determines the wetting temperature $k_{B} T_{w} \approx 1.142 \varepsilon$. We further use Young's equation (1) to determine the temperature dependence of the contact angle $\theta(T)$, as shown in Fig. 4 These results can be compared with those obtained from cDFT that is given by Eq. (24). The resulting two-dimensional density profiles for several representative temperatures are displayed in Fig. 5 (first row).

\section{B. Wetting properties of corrugated walls}

We now turn our attention to the wetting properties of corrugated walls. To this end, we fix the width of each pillar at $L_{1}=0.5 \sigma$ and consider three different pillar heights $(D=0.5 \sigma, D=\sigma$ and $D=2 \sigma)$ and two periodicities $(L=2 \sigma$ and $L=4 \sigma)$. Somewhat naively, we can assign a roughness parameter value to each of the substrate models as follows:

$$
r \equiv 1+2 D / L
$$

and search for the correlation between $r$ and the surface wettability. Note that this simple definition of the roughness parameter is somewhat arbitrary and that alternative possibilities how the define surface roughness are available [41, 42. First, we fix the temperature at $k_{B} T=1.1 \varepsilon\left(T=0.78 T_{c}\right)$, which is below the wetting temperature for the planar wall. From Table 1, where we show the contact angle for each substrate, we find that: i) somewhat surprisingly, the contact angles of different models with identical $r$ almost coincide, which supports the definition (26), and ii) the contact angle increases with $r$. For relatively high $r$, the increase in the apparent angle is sufficiently pronounced, such that the hydrophilic surface $(\theta<\pi / 2)$ becomes hydrophobic $\left(\theta^{*}>\pi / 2\right)$. Note that these observations are in direct contradiction with Wenzel's law given by Eq. (2).

Next, we consider a temperature dependence of the contact angle. The most important conclusion that can be drawn from the results shown in Fig. 6 is that corrugation always decreases the hydrophilicity of the wall. More specifically, for relatively low corrugation (small $r$ ), the surface roughness shifts the wetting temperature to significantly higher values than of the planar wall. For more strongly corrugated walls, the role of the roughness is even more dramatic as the walls become non-wetting. The models with the roughness parameter $r=2$ become hydrophobic over almost the entire range of temperatures apart from the immediate vicinity of the critical temperature where $\theta \approx 90^{\circ}$. Most notably, when the surface effects become sufficiently strong (corresponding to the model with the highest roughness parameter), the contact angle increases with the temperature, which ultimately leads to drying at a temperature corresponding to $k_{B} T_{d} \approx 1.24 \varepsilon$.

These results can be compared with representative two-dimensional density profiles that are obtained using cDFT (see Fig. 5p to produce the states of the sessile drop for the same substrates as considered in Fig. 6. Here, we present the equilibrium profiles corresponding to relatively large systems with a lateral and a normal size of $60 \sigma$ and $20 \sigma$, respectively. We have also tested our results for different system sizes, different total amounts of fluid molecules and different initial states. Since for our model the wetting transition is first order, there is a free-energy barrier between partial and complete wetting states. In order to avoid a situation where the system is trapped in a local minimum of the free energy, we have started from two distinct initial configurations: one corresponding to a partial wetting $(\theta>0)$ state and one corresponding to a complete wetting $(\theta=0)$ state, and considered the one with a lower value of the free energy. We observe that the hydrophilic nature of the substrates de- 

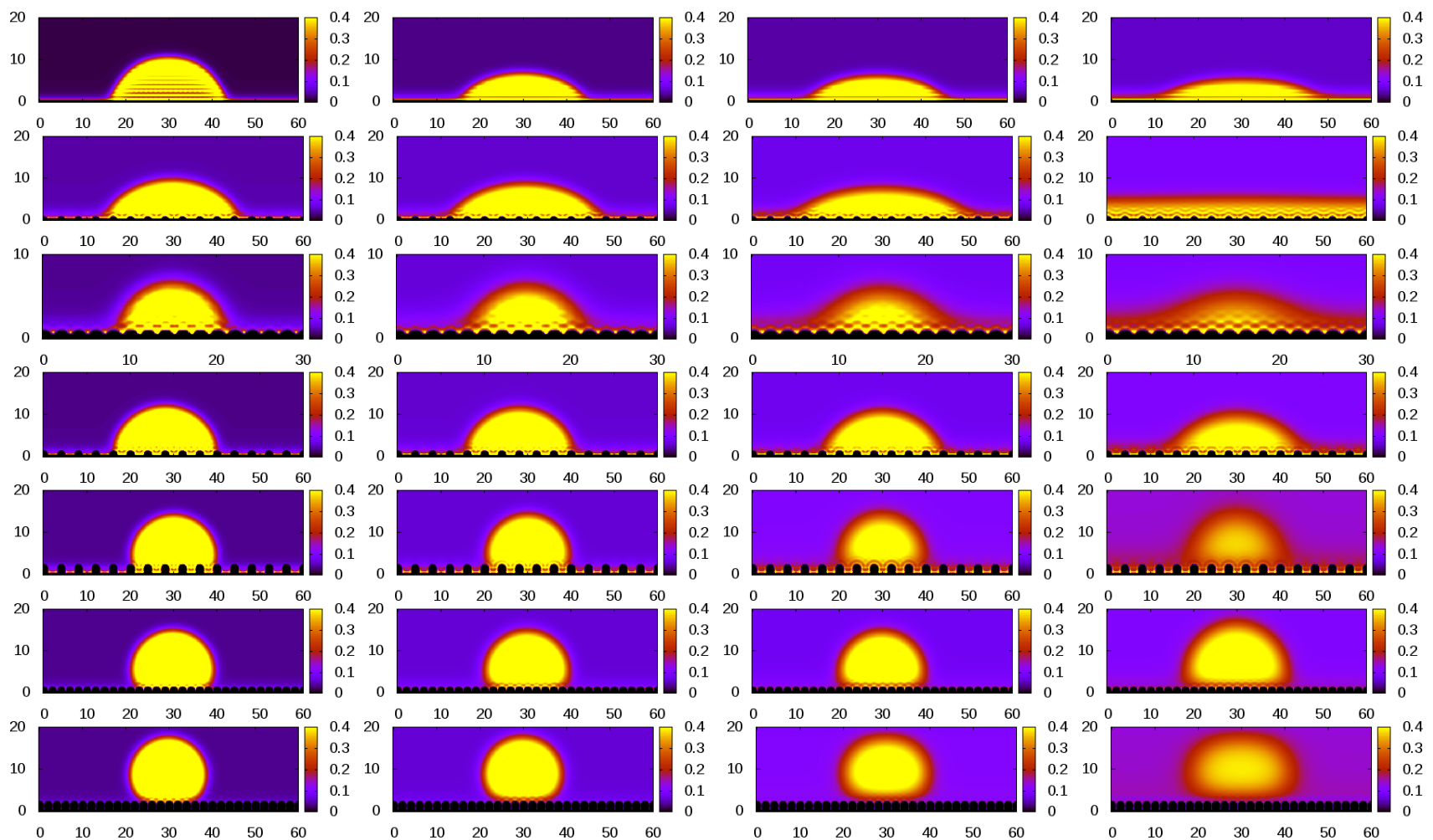

FIG. 5: Two-dimensional density profiles of a sessile drop on walls of different corrugations. First row: Case 1 (smooth wall); the profiles correspond to the temperatures (from left to right): $k_{B} T / \varepsilon=1, k_{B} T / \varepsilon=1.1, k_{B} T / \varepsilon=1.12$ and $k_{B} T / \varepsilon=1.14$. Second row: Case 2; the profiles correspond to the temperatures (from left to right): $k_{B} T / \varepsilon=1.1, k_{B} T / \varepsilon=1.15, k_{B} T / \varepsilon=1.2$ and $k_{B} T / \varepsilon=1.25$. Third row: Case 3; the profiles correspond to the temperatures (from left to right): $k_{B} T / \varepsilon=1.1$, $k_{B} T / \varepsilon=1.2, k_{B} T / \varepsilon=1.25$ and $k_{B} T / \varepsilon=1.28$. Fourth row: Case 4; the profiles correspond to the temperatures (from left to right): $k_{B} T / \varepsilon=1.1, k_{B} T / \varepsilon=1.15, k_{B} T / \varepsilon=1.18$ and $k_{B} T / \varepsilon=1.2$. Fifth row: Case 5; the profiles correspond to the temperatures (from left to right): $k_{B} T / \varepsilon=1.1, k_{B} T / \varepsilon=1.2, k_{B} T / \varepsilon=1.3$ and $k_{B} T / \varepsilon=1.35$. Sixth row: Case 6; the profiles correspond to the temperatures (from left to right): $k_{B} T / \varepsilon=1.1, k_{B} T / \varepsilon=1.15, k_{B} T / \varepsilon=1.18$ and $k_{B} T / \varepsilon=1.2$. Seventh row: Case 7; the profiles correspond to the temperatures (from left to right): $k_{B} T / \varepsilon=1.1, k_{B} T / \varepsilon=1.2, k_{B} T / \varepsilon=1.3$ and $k_{B} T / \varepsilon=1.35$. In all cases, the equilibrium density distributions correspond to systems of dimensions $60 \sigma \times 20 \sigma$ and are expressed in units of $\sigma$ and $\varepsilon$. Each case corresponds to a substrate model according to Table 1.

creases with the roughness parameter which is consistent with the results that are obtained using the grand canonical DFT. In particular, while for $r=1.25-1.5$ the liquid tends to spread over the solid surface as the temperature increases and for $r=2$ the contact angle is high and nearly constant, there is an increase in the contact angle of the liquid drop for $r=3$ as the temperature increases. From a macroscopic perspective, the case shown in the lowest row of Fig. 5 provides a so called Cassie-Baxter (or fakir) state, where the drop sits on top of the surface with the vapor trapped underneath, even though the material of the wall is hydrophilic; this situation occurs for the temperatures $k_{B} T / \varepsilon=1.1$ and $k_{B} T / \varepsilon=1.2$. At the higher temperatures the liquid drop already "levitates" above the wall, in agreement with the results shown in Fig. 6 .

Finally, we consider a substrate with a surface of alternating smooth and corrugated regions of equal areas. For the corrugated section, we use a model with $D=0.5 \sigma$, $L_{1}=0.5 \sigma$ and $L=2 \sigma$ (case 3). In Fig. 7, we dis- play the equilibrium density profiles that are obtained by cDFT. At higher temperatures, the liquid separates into cylindrically shaped structures of identical cross sections above the smooth parts of the surface, while the corrugated parts remain dry. However, when the temperature is lowered sufficiently, the liquid undergoes a morphological transition and forms a single bulge. This transition can be explained in terms of the competition between the dewetting of the rough section of the wall by the liquid and the energetic cost of creating a liquid-vapor interface, which is high at low temperatures.

These results are complemented by a standard grand canonical DFT calculation, with an ambient phase of saturated vapor, see Fig. 8 . For a temperature below the wetting temperature of the corresponding planar wall $T_{w}$, only a microscopic layer of liquid forms at the smooth sections and impregnates the tiny grooves in the rough parts. Above $T_{w}$ but below the wetting temperature of the corrugated section $T_{w}^{r}$, cylindrical segments of liquid form at the smooth sections of the surface, which cor- 


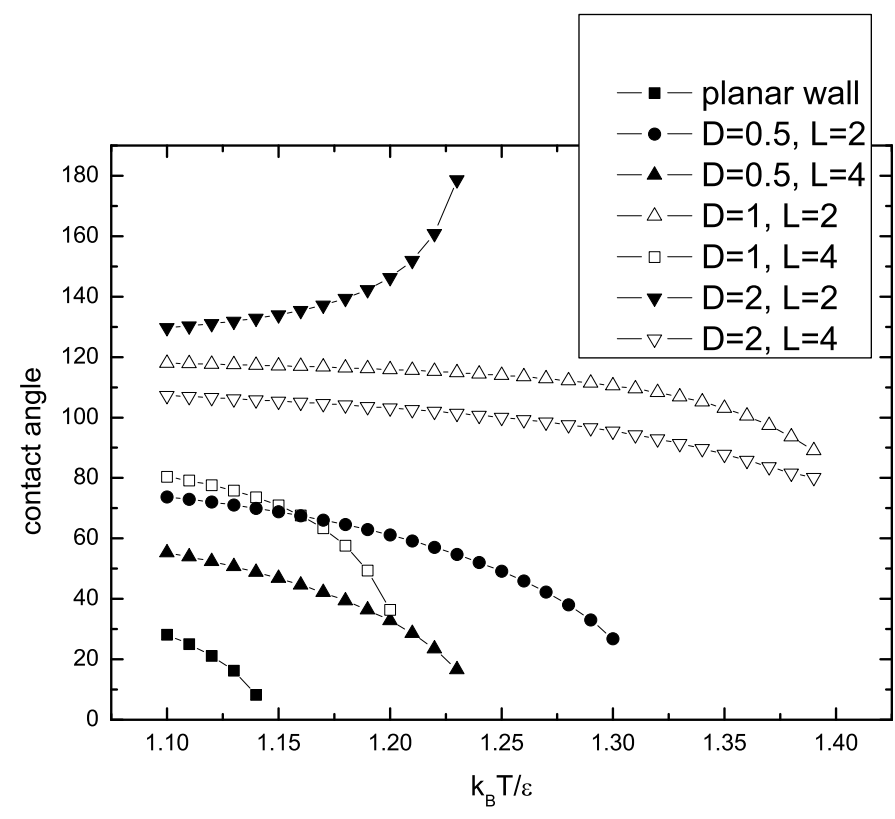

FIG. 6: Comparison of the temperature dependence of the contact angle (in degrees) for walls with different types of corrugation.

responds to the high-temperature state shown in Fig. 7. Finally, the entire surface is completely wet above $T_{w}^{r}$. Note that although the liquid-vapor interface is flat, and thus does not reflect the shape of the wall, the lateral inhomogeneity in the liquid structure remains fairly pronounced near the wall.

\section{SUMMARY OF THE RESULTS}

In this paper, we have studied the wetting properties of microscopically rough surfaces of hydrophilic material. The methods used in the study and the main results are summarized below:

- We have applied a density functional theory that is based on the Tarazona tensorial version of FMT, which is most likely the most accurate microscopic approach available for non-uniform fluids. This FMT-DFT avoids the spurious divergencies produced by the original Rosenfeld FMT version, satisfies exact statistical mechanical sum rules and provides correct limits in reduced dimensions.

- We have started with a detailed description of a "reference system" of an ideally planar wall interacting with the fluid particles via long-ranged dispersion forces. The wall exhibits a first-order wetting transition (well below the critical temperature, $\left.T_{w} / T_{c}=0.8\right)$ that can be characterized as the temperature at which a macroscopically thick liquid layer forms at the wall or, equivalently, as the temperature at which the contact angle of a sessile drop

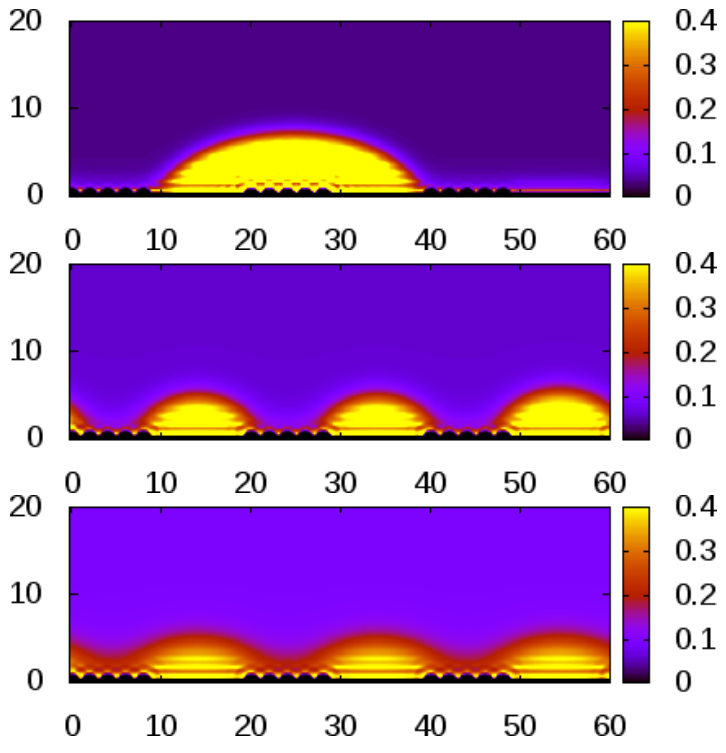

FIG. 7: Two-dimensional density profiles on a wall where smooth and rough parts of the surface alternate. Each rough part is made out of five steps with corrugation parameters $D=0.5 \sigma$ and $L=2 \sigma$. The equilibrium profiles are obtained from a constrained DFT and correspond to the temperatures (from top to bottom): $k_{B} T / \varepsilon=1.1, k_{B} T / \varepsilon=1.2$ and $k_{B} T / \varepsilon=1.3$. The equilibrium density distributions correspond to systems of dimensions $60 \sigma \times 20 \sigma$ and are expressed in units of $\sigma$ and $\varepsilon$.

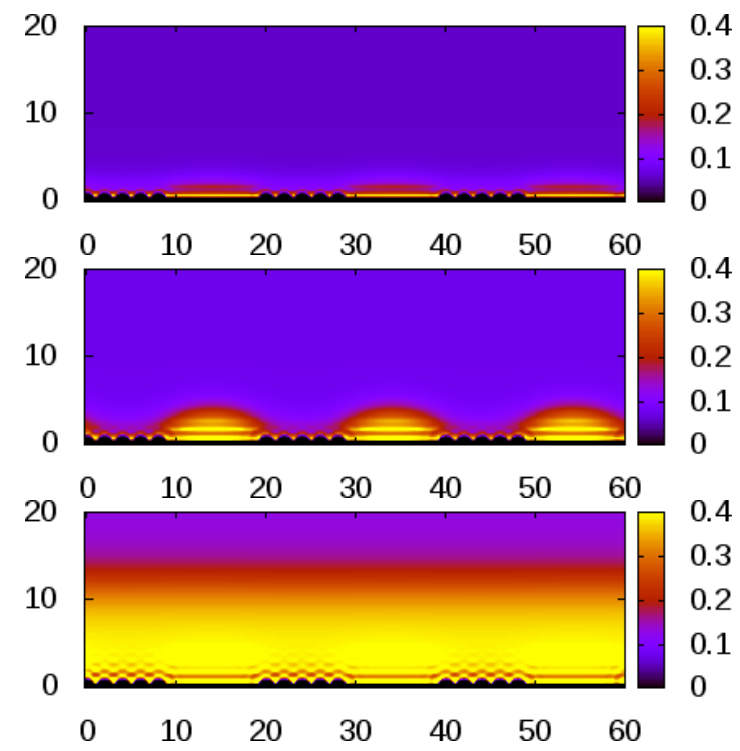

FIG. 8: Two-dimensional density profiles on a wall where smooth and rough parts of the surface alternate. Each rough part is made out of five steps with corrugation parameters $D=0.5 \sigma$ and $L=2 \sigma$. The equilibrium profiles are obtained from a grand canonical ensemble DFT and correspond to the temperatures (from the top to the bottom): $k_{B} T / \varepsilon=1.1$, $k_{B} T / \varepsilon=1.2$ and $k_{B} T / \varepsilon=1.35$. These results should be compared with figure 6] The equilibrium density distributions correspond to systems of dimensions $60 \sigma \times 20 \sigma$ and are expressed in units of $\sigma$ and $\varepsilon$. 


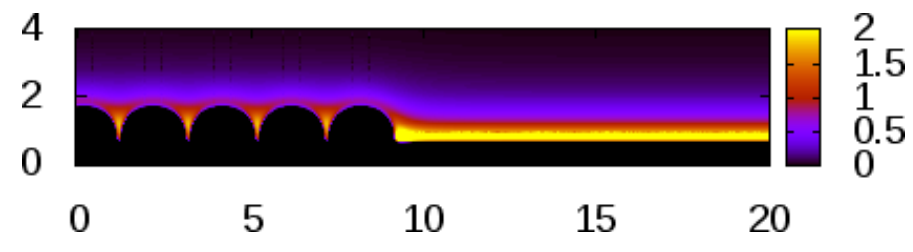

FIG. 9: Modulus of the external potential (expressed in units of $\varepsilon$ ) of a substrate consisting of $\operatorname{rough}\left(D=\sigma, L_{1}=0.5 \sigma\right)$ and smooth sections in the $x-z$ plane; the plot shows that the presence of the pillar effectively weakens the net potential of the wall except inside the grooves. The coordinates correspond to units of $\sigma$.

vanishes. We have used both fixed- $\mu$ and fixed- $\langle N\rangle$ DFT to determine $T_{w}$ and the temperature dependence of the contact angle.

- We have found that microscopic surface roughness always deteriorates the wall wettability. This result is in direct contradiction with the classical Wenzel's law, which predicts that the wetting properties of the wall are always amplified by surface corrugation, such that the hydrophilicity of surfaces increases with surface roughening. We discuss this point below.

- For a liquid deposited on substrates with alternating flat and rough sections we observed two possible morphologies depending on the temperature. At high temperatures, the liquid tends to wet the planar sections of the wall and forms periodic cylindrical segments within which the rough sections remain dry. However, as the temperature decreases, a configuration with only one liquid bulge is favored, in view of the high energetic cost of creating a liquid-vapor interface.

\section{CONCLUDING REMARKS}

The main conclusion of this study is that DFT results of wetting properties of microscopically rough surfaces contradict macroscopic predictions, such as those implied by Wenzel's law. To understand this point we briefly revisit the arguments leading to Eq. (2): assuming the liquid-wall interface beneath a drop deposited on a rough surface follows the wall corrugation, we minimize the surface energy for a virtual displacement $\mathrm{d} x$ of the contact line

$$
\mathrm{d} E=r\left(\gamma_{s l}-\gamma_{s v}\right) \mathrm{d} x+\gamma \mathrm{d} x \cos \theta^{*} .
$$

Within this picture the effect of the surface roughness only manifests as an increase in the substrate area by a factor of $r$, whereas the other aspects of surface corrugation are ignored. To interpret our DFT results, we now present the meso- and microscopic implications of the non-planar wall geometry. We consider nearly flat wall with ideal planarity that is perturbed by adding (or removing) a microscopically small portion of the material at some point on (from) its surface. The perturbation can be represented by a single pillar (or a single groove) that is deposited (etched) on (into) the wall, although the specific shape of the barrier (well) is not crucial. More importantly, the mean height of the liquid-vapor interface is now a function of the horizontal position $\ell=\ell(x)$. To be specific, we may consider a complete wetting regime, such that the pressure is slightly below its saturation value. If the planar wall is perturbed, the liquid-vapor interface must bend around the barrier, which costs an additional local bending energy per unit area, $\delta E \approx \gamma / 2(\nabla \ell)^{2}$; thus the wall barrier also acts as an energy barrier. This effect is also responsible for the binding of the local interface near the edge of an apex-shaped substrate [29, 30, and for the finite thickness of a wetting layer on a spherical wall [43 45], even in the limit of bulk coexistence.

Let us now consider a semi-infinite rough surface $(D=$ $\left.\sigma, L_{1}=0.5 \sigma\right)$ and compare the magnitude of the wall potential with that corresponding to a semi-infinite smooth surface, as shown in Fig. 9. We can immediately draw two conclusions: i) Unlike the potential for the smooth section, the potential above the rough surface exhibits lateral inhomogeneity, although weakly. The shape of the adsorbed film tends to follow the geometry of the external field; therefore, adsorption at the rough surface is more energetically expensive because of the aforementioned cost of the surface tension. ii) More importantly, the excluded volume effects from the presence of the pillars produce a region that is inaccessible to the fluid molecules, that have a significantly larger volume than the actual volume of the pillars. Note that as an effect the rectangular pillars appear rounded. Consequently, the external field above the pillars is markedly lowered relative to the smooth surface, which explicitly demonstrates why surface roughness hinders adsorption. On the other hand, there is a fairly strong field within the accessible volume between the pillars, i.e., inside the grooves. Therefore, it is much easier for the liquid to fill the grooves than wet the structured surfaces, which is completely in agreement with the macroscopic arguments [3].

Finally, it is important to stress out that on a macroscopic scale the wall geometry does indeed promote fluid adsorption. A simple example of this is a wedge-like cavity, the geometry of which enhances the adsorption and decreases the effective contact angle. In a wedge with a tilt angle $\alpha$ the roughness parameter is $r=\sec \alpha$. For $\theta<\alpha$ the wedge is completely filled $\left(\theta^{*}=0\right)$ even though the side walls are partially wet [46 49 and thus 
the wedges induce the filling transition at which a macroscopic amount of liquid occurs at a temperature $T_{f}<T_{w}$. This is in a complete qualitative accord with Wenzel's law. However, such a scenario is only conceivable if the wedge-like structure has macroscopic dimensions which is certainly not the case of our corrugation model. From this it follows that there must exist a crossover between a microscopic scale, where the surface structure suppresses fluid adsorption, and a macroscopic scale, where the adsorption is enhanced by the wall geometry. This, we believe, is an important point in terms of the modern trends in engineering branches to apply phenomenological theories on nanoscopically small systems (so called nanothermodynamics). The danger of these attempts has been clearly exemplified here.

We stress here once again that the choice to represent the macroscopic treatment of rough surfaces by Eq. (2) was rather arbitrary and that Wenzel's law plays no prominent role within the class of phenomenological theories. The only specific feature of Wenzel's law is that it predicts that the surface roughness lowers the wetting temperature, i.e. that $\theta^{*}$ may vanish even though $\theta>0$. Note that this prediction is not supported by the experiments on textured surfaces [50] which revealed that the wetting (or drying) temperature is unaffected by the surface roughness. This is because, in the hydrophilic case, the Wenzel's regime fails to describe a propagation of liquid film in the solid grooves (so-called hemi-wicking [3, 32]). If this second phenomenon is taken into account it follows that $\theta^{*}=0$ only if $\theta=0$ but even in this regime the surface wettability is improved $\left(\theta>\theta^{*}>0\right)$. This still contradicts our microscopic results, however.

In this work, we dealt with a class of very simple models of geometrically nonuniform surfaces and of course further investigations in this direction are needed. One cannot exclude that the picture of wetting on more re- alistic model surfaces is more complex than that made in this work. This study can be most directly extended in several ways. Fixed molecular parameters have been considered throughout this study and only the wall geometry and temperature were allow to vary. In particular, the amplitudes of the wall-fluid and fluid-fluid interactions were assumed to be identical. It would be interesting to investigate whether our conclusions remain unchanged if the wall potential strength is allowed to vary. One can speculate, that for $\varepsilon_{w}<\varepsilon$, i.e., a weak substrate, the liquid would penetrate into the wall pockets effectively smoothing out the surface, which, as a result, would become a stronger adsorbent than the solid planar wall. On the other hand, for an appreciably stronger wall potential than that used in our model, a sequence of layering transitions may occur; it would then be interesting to determine whether the interfaces between the neighboring layers are planar or whether their structure mirrors the wall geometry. Extending the fluid model to non-spherical bodies, such as model polymers or liquid crystals, would be challenging and computationally demanding, and has only been attempted rather recently [51. Finally, it would be interesting, especially in view of the results presented in Figs. 8 and 7 , to investigate the dynamical aspects of the model and to track the system evolution from a particular initial state toward equilibrium.

\section{Acknowledgments}

I acknowledge the financial support from the Czech Science Foundation, Grant No. 13-09914S.
[1] P. G. de Gennes, F. Brochard-Wyart, and D. Quéré, Capillarity and Wetting Phenomena: Drops, Bubbles, Pearls, Waves, Springer, New York (2004).

[2] J. N. Israelachvilli, Intermolecular and Surface Forces, Academic Press, San Diego (2011).

[3] D. Quéré, Physica A 313, 32 (2002).

[4] T. Young, Philos. Trans. R. Soc. London 95, 65 (1805).

[5] R. Fürstner, W. Barthlott, C. Neinhuis, and P. Walzel, Langmuir 21, 956 (2005).

[6] K. Koch, B. Bhushan, and W. Barthlott, Soft Matter 4, 1943 (2008).

[7] S. Minko, Polymer Rev. 46 , 397 (2006).

[8] S. Y. Chou, P. R. Krauss, and P. J. Renstrom, Science 272, 85 (1996).

[9] R. N. Wenzel, Ind. Eng. Chem. 28, 988 (1936).

[10] S. Dietrich, in Phase Transitions and Critical Phenomena, edited by C. Domb and J. L. Lebowitz (Academic, New York, 1988), Vol. 12.

[11] D. E. Sullivan and M. M. Telo da Gama, in Fluid Interfacial Phenomena, edited by C. A. Croxton (Wiley, New
York, 1985).

[12] M. Schick, in Liquids and Interfaces, edited by J. Chorvolin, J. F. Joanny, and J. Zinn-Justin (Elsevier, New York, 1990).

[13] D. Bonn, J. Eggers, J. Indekeu, J. Meunier, and E. Rolley, Rev. Mod. Phys. 81, 739 (2009).

[14] P. Tarazona and R. Evans, Mol. Phys. 48, 799 (1983).

[15] P. Bryk, W. Rżysko, A. Malijevský, and S. Sokołowski, J. Colloid Interface Sci. 313, 41 (2007).

[16] O. Pizio, H. Dominguez, L. Pusztai, and S. Sokolowski, Physica A 388, 2278 (2009).

[17] E. Ruckenstein and G. O. Berim, Adv. Colloid Interface Sci. 157, 1 (2010).

[18] G. O. Berim and E. Ruckenstein, Langmuir 28, 11384 (2012).

[19] F. Dutka, M. Napiórkowski, and S. Dietrich, J. Chem. Phys. 136 , 064702 (2012).

[20] D. Zhou, J. Mi and C. Zhong, J. Phys. Chem. C 116, $14100(2012)$

[21] S. K. Das and K. Binder, Europhys. Lett. 92, 26006 
(2010).

[22] V. Kumar, S. Sridhar, and J. R. Errington, J. Chem. Phys. 135, 184702 (2011).

[23] D. Seveno, T. D. Blake, and J. De Connick, Phys. Rev. Lett. 111, 096101 (2013).

[24] R. Evans, Adv. Phys. A 28, 143 (1979).

[25] P. Tarazona, Phys. Rev. Lett. 84, 694 (2000).

[26] P. Tarazona, Physica A 306, 243 (2002).

[27] Y. Rosenfeld, Phys. Rev. Lett. 63, 980 (1989).

[28] A. Malijevský, J. Phys.: Condens. Matter 25, 445006 (2013).

[29] A. O. Parry, M. J. Greenall, and J. M. Romero-Enrique, Phys. Rev. Lett. 90, 046101 (2003).

[30] A. Malijevský, J. Phys.: Condens. Matter 26, 315002 (2014).

[31] G. Wolansky and A. Marmur, Colloids Surf. A 156, 381 (1999).

[32] J. Bico, U. Thiele, and D. Quéré, Colloids Surf. A 206, 41 (2002).

[33] C. Ishino and K. Okumura, Eur. Phys. J. E 25, 415 (2008).

[34] D. Quéré, Annu. Rev. Mater. Res. 38, 71 (2008).

[35] A. Malijevský, A. O. Parry, J. Phys.: Condens. Matter 25, 305005 (2013).

[36] P. Tarazona, J. A. Cuesta and Y. Martinez-Raton, in A. Mulero (Ed.), Theory and Simulation of Hard-Sphere Fluids and Related Systems, Lect. Notes Phys. 753 (Springer, Berlin Heidelberg 2008), pp. 247-382.

[37] J. A. Hernando and L. Blum, J. Phys.: Condens. Matter 13, L577, (2001).
[38] A. González, J. A. White, F. L. Romaán, S. Velasco, and R. Evans, Phys. Rev. Lett. 79, 2466 (1997).

[39] A. González, J. A. White, F. L. Román, and R. Evans, J. Chem. Phys. 109, 3637 (1998).

[40] J. A. White, A. Gonzáles, F. L. Román, and S. Velasco, Phys. Rev. Lett. 84, 1220 (2000).

[41] D. Whitehouse, Surfaces and their Measurement, (Boston: Butterworth-Heinemann 2012).

[42] E. S. Gadelmawla, M. M. Koura, T. M. A. Maksoud, I. M. Elewa, H. H. Soliman, J. Mater. Process. Tech. 123, 133 (2002).

[43] T. Bieker and S. Dietrich, Physica A 252, 85 (1998).

[44] M. C. Stewart and R. Evans, Phys Rev. E 71, 011602 (2005).

[45] A. Nold, A, Malijevský, and S. Kalliadasis, Phys. Rev. E 84, 021603 (2011).

[46] K. Rejmer, S. Dietrich, and M. Napirkówski, Phys. Rev. E 60, 4027 (1999).

[47] A. O. Parry, C. Rascón, and A. J. Wood, Phys. Rev. Lett. 85, 345 (2000).

[48] A. Milchev, M. Müller, K. Binder, and D. P. Landau, Phys. Rev. Lett. 90, 136101 (2003).

[49] A. Malijevský and A. O. Parry, Phys. Rev. Lett. 110, 166101 (2013).

[50] S. Shibuichi, T. Onda, N. Satoh, and K. Tsujii, J. Phys. Chem 100, 19512 (1996).

[51] P. Patrício, J. M. Romero-Enrique, N. M. Silvestre, N. R. Bernardino, and M. M. Telo Da Gama, Mol. Phys. 109, 1067 (2011). 